\title{
Trades and defining sets: theoretical and computational results
}

\author{
Colin Ramsay
}

Given a particular combinatorial structure, there may be many distinct objects having this structure. When investigating these, two natural questions to ask are:

$\triangleright$ Given two objects, where and how do they differ?

$\triangleright$ How much of an object is necessary to uniquely identify it?

These two questions are obviously related, with the first leading to the concept of a trade, and the second to that of a defining set. In this thesis we study trades and defining sets, in the context of $t-(v, k, \lambda)$ designs. In our enquiries, we make use of both theoretical and computational techniques, with theoretical results leading to more efficient programmes and computational results suggesting lines of theoretical enquiry.

We investigate the spectrum of trades, and prove an extant conjecture regarding this [1]. Our results also suggest a more general version of this conjecture [2]. A $t-(v, k, \lambda)$ design where $\lambda=1$ is called a Steiner design, and the related trades are called Steiner trades. In the case $t=2$, we establish the spectrum of Steiner trades for each value of $k$, except for a finite number of values in each case $[3,4]$.

The connection between trades and defining sets is used to obtain some new theoretical results on defining sets of designs, and is exploited throughout the thesis $[5,6]$. We also consider the collections of all trades and all defining sets in a design.

A simple design is one which is a set, as opposed to a multiset. We present an algorithm to enumerate all the trades in simple designs. For non-simple designs we introduce the concept of a discriminating set, and present an algorithm to enumerate these. Output from these algorithms was used to investigate the trades and defining sets of a number of designs. An error in a published result was discovered, and some new results were obtained [7].

Given part of a design, its completions are all those designs that contain it. An existing algorithm to complete partial designs is examined, and a heuristic yielding a much improved algorithm for Steiner designs is discussed. This completion routine was used to investigate a number of designs, and new information on the size and distribution of their defining sets was obtained [8].

Received 5th November, 1998

Thesis submitted to The University of Queensland, February 1998. Degree approved, August 1998. Supervisors: Dr. G. Havas and Professor A.P. Street.

Copyright Clearance Centre, Inc. Serial-fee code: 0004-9729/99 \$A2.00+0.00. 


\section{REFERENCES}

[1] B.D. Gray and C. Ramsay, 'On a conjecture of Mahmoodian and Soltankhah regarding the existence of $(v, k, t)$ trades', Ars Combin. 48 (1998), 191-194.

[2] B.D. Gray and C. Ramsay, 'On the spectrum of $[v, k, t]$ trades', J. Statist. Plann. Inference 69 (1998), 1-19.

[3] B.D. Gray and C. Ramsay, 'On the spectrum of Steiner $(v, k, t)$ trades (I)', J. Combin. Math. Combin. Comput. (to appear).

[4] B.D. Gray and C. Ramsay, 'On the spectrum of Steiner $(v, k, t)$ trades (II)', Graphs Combin. (to appear).

[5] B.D. Gray and C. Ramsay, 'Some results on defining sets of $t$-designs', Bull. Austral. Math. Soc. 59, 203-215.

[6] C. Ramsay, 'On a family of $S T S(v)$ whose smallest defining sets contain at least $b / 4$ blocks', Bull. Inst. Combin. Appl. 20 (1997), 91-94.

[7] C. Ramsay, 'An algorithm for enumerating trades in designs, with an application to defining sets', J. Combin. Math. Combin. Comput. 24 (1997), 3-31.

[8] C. Ramsay, 'An algorithm for completing partials, with an application to the smallest defining sets of the STS(15)', Utilitas Math. 52 (1997), 205-221.

Centre for Discrete Mathematics and Computing

Department of Computer Science and Electrical Engineering

The University of Queensland

Queensland 4072

Australia

e-mail: cram@csee.uq.edu.au 